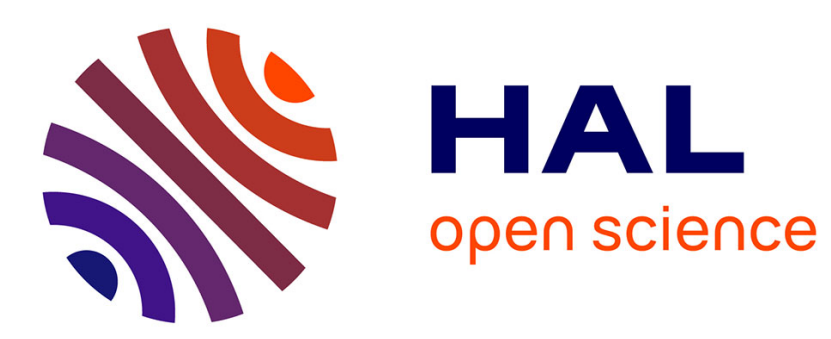

\title{
On the effect of the local overall interaction on the postbuckling of uniformly compressed channels
}

\author{
Marcello Pignataro, Angelo Luongo, Nicola Rizzi
}

\section{To cite this version:}

Marcello Pignataro, Angelo Luongo, Nicola Rizzi. On the effect of the local overall interaction on the postbuckling of uniformly compressed channels. Thin-Walled Structures, 1985, 3 (4), pp.293-321. hal-00788743

\section{HAL Id: hal-00788743 \\ https://hal.science/hal-00788743}

Submitted on 15 Feb 2013

HAL is a multi-disciplinary open access archive for the deposit and dissemination of scientific research documents, whether they are published or not. The documents may come from teaching and research institutions in France or abroad, or from public or private research centers.
L'archive ouverte pluridisciplinaire HAL, est destinée au dépôt et à la diffusion de documents scientifiques de niveau recherche, publiés ou non, émanant des établissements d'enseignement et de recherche français ou étrangers, des laboratoires publics ou privés. 


\section{On the Effect of the Local Overall Interaction on the Postbuckling of Uniformly Compressed Channels}

\section{Marcello Pignataro, Angelo Luongo and Nicola Rizzi}

Istituto di Scienza delle Costruzioni-Università di Roma,

Via Eudossiana 18, 00184 Roma, Italy

\section{ABSTRACT}

On the basis of the general theory of elastic stability due to Koiter, postbuckling analysis of simply supported channels under uniform compression is performed. Attention is essentially focused on locall eulerian and local/flexural-torsional simultaneous buckling modes interaction. The column is treated as a plate assemblage. Linearized expressions for the displacement field are employed while assuming strain-displacements relationships that are linear for the curvatures and up to second order terms for the in-plane strains. The total potential energy is hence written up to third order terms in order to investigate asymmetric buckling phenomena. A discrete model is developed through an automatic procedure of algebraic manipulation, and an extensive parametric analysis is performed. After determining the range of geometric parameters which characterize different types of interaction, it is found that in the postbuckling range the local/eulerian interaction is more dangerous than the local/flexural-torsional one, due to the column higher imperfection sensitivity.

\section{INTRODUCTION}

Modern metal structures such as aeronautical, ship-building and civil engineering constructions, are characterized by the use of thin plates of high strength steels. Such structural plate elements are, however, likely to buckle locally in compression, bending or shear. Accordingly, local plate buckling considerations are of great importance in the design of thin 
plated structures. In addition, when thin plates are utilized as component plate elements of thin-walled stiffened and unstiffened compression members, local plate buckling may interact with overall buckling. Local buckling assumes that the line junctions between intersecting plates remain straight and the cross-sectional shape of the member undergoes distortion. Overall buckling is characterized by no distortion of the crosssection in its plane.

The occurrence of simultaneous or nearly simultaneous buckling modes can produce an adverse effect on the strength in that, structures with stable postbuckling behaviour by correspondence with each of the single buckling modes may exhibit imperfection sensitivity when two (or more) buckling modes interact.

The interaction behaviour of thin-walled structural elements in compression has received a great deal of attention in the past years by many researchers. Among the pioneering works we wish to mention Grimaldi and Pignataro ${ }^{1}$ who analysed the eulerian/flexural-torsional interaction and Van der Neut ${ }^{2}$ and Graves-Smith ${ }^{3}$ who first studied the interaction between local and Euler buckling in thin-walled compression members by using mathematical models which included the nonlinear membrane stiffness of the plates forming the columns. In more recent years analytical investigation has been replaced by numerical methods, among which are the most competitive one for prismatic structures subjected to end loads seems to be the finite strip method. Graves-Smith and Sridharan ${ }^{4.5}$ have used this method extensively by furnishing versions particularly suitable for post-locally-buckled analysis of plate structures within the frame of a perturbation procedure.

In a few recent works, interaction analysis is based on the assumption that, under increasing loading, the column undergoes local buckling first, followed by overall deflection at higher loading level. Due to weakening effects of local buckling, the overall deflection takes place at lower load than the column would carry in the absence of the local buckling. In a number of papers Hancock ${ }^{6,7}$ and Bradford and Hancock ${ }^{8}$ investigated the reduction of the effective flexural resistances and the associated overall buckling loads through a nonlinear analysis of the column in the post-locally-buckled range. By adopting the finite strip method they solved the ensuing equations by the Newton-Raphson method, and the results were compared to experimental tests. A somewhat parallel. analysis, based on the empirical formulas to evaluate the effective plates" width, was performed by Wang and Pao. ${ }^{9}$ They analysed a channel under 
compression by using the finite element method within an iterative procedure. Rhodes and Harvey, ${ }^{10}$ Loughlan and Rhodes, ${ }^{11}$ Loughlan ${ }^{12}$ and Upadhya and Loughlan ${ }^{13.14}$ investigated the post-locally-buckled behaviour of a plain and lipped channel under compression without and with local or overall imperfections. The Rayleigh-Ritz method was used to obtain local buckling loads and a semi-energy method is employed to describe postbuckling interaction behaviour.

A different approach was used by Benito ${ }^{15}$ and Sridharan and Benito $^{16.17}$ who employed the finite strip method within the frame of Koiter general theory of stability. They analysed nearly simultaneous buckling modes in the presence of initial imperfections and solved by Newton-Raphson method for the relevant nonlinear equilibrium equations in terms of the displacement parameters for a number of sections.

In this paper the effect of simultaneous buckling modes on the postbuckling behaviour of uniformly compressed channels simply supported at the ends is investigated on the basis of Koiter theory of elastic stability. ${ }^{18}$ The column is considered to be a plate assemblage. Initial imperfections are not considered and bifurcation corresponding to the two simultaneous buckling modes local/flexural and local/flexuraltorsional is analysed. The total potential energy is expanded up to third order terms in order to obtain the slope to the bifurcated paths. The displacement field is discretized through adequate shape functions, which account for compatibility along the plate junctions and boundary conditions at the free longitudinal edges and at the beam ends. The relevant perturbation equations are obtained by means of an automatic procedure of algebraic manipulation. Results show that interaction between local and overall buckling has an adverse effect on column strength and it is far more detrimental for the local/eulerian interaction than for the local/ flexural-torsional one. In particular, results regarding the local/flexuraltorsional coupling coincide with those ones furnished in ref. 16 when the number of local buckling halfwaves is sufficiently large. In this case the slope of all bifurcated paths approaches zero.

\section{BUCKLING AND POSTBUCKLING ANALYSIS}

In this section we present a brief sketch of Koiter nonlinear bifurcation theory in the form essentially due to Budiansky. ${ }^{19}$ 
Let us consider a hyperelastic body system subjected to conservative loads characterized by a total potential energy functional $\Phi[\mathbf{u} ; \lambda]$, where $\mathbf{u}$ is the displacement field and $\lambda$ a parameter governing the external force field acting on it. The equilibrium condition is obtained by requiring the functional $\Phi[\mathbf{u} ; \lambda]$ to be stationary with respect to kinematically admissible displacement fields, that is

$$
\Phi^{\prime}[\mathbf{u} ; \lambda] \delta \mathbf{u}=0 \quad \forall \delta \mathbf{u}
$$

where a prime denotes Fréchet differentiation with respect to $u$. Equation (1) furnishes all relations between $\boldsymbol{u}$ and $\lambda$ which we shall call equilibrium paths $\mathbf{u}=\mathbf{u}(\lambda)$.

In buckling problems it is assumed that at a certain critical value $\lambda_{c}$ of the load factor $\lambda$, the state $\mathbf{u}_{c}$ belongs to two different equilibrium paths: the fundamental one, $\mathbf{u}^{\mathrm{f}}(\lambda)$, which is assumed to be known, and the bifurcated path $\mathbf{u}^{\mathrm{b}}(\lambda)$

$$
\begin{aligned}
& \mathbf{u}=\mathbf{u}^{\mathrm{f}}(\lambda) \\
& \mathbf{u}=\mathbf{u}^{\mathrm{b}}(\lambda) \\
& \mathbf{u}^{\mathrm{f}}\left(\lambda_{c}\right)=\mathbf{u}^{\mathrm{b}}\left(\lambda_{c}\right)=\mathbf{u}_{c}
\end{aligned}
$$

Let us now introduce the differential state variable

$$
\mathbf{v}(\lambda)=\mathbf{u}^{b}(\lambda)-\mathbf{u}^{f}(\lambda)
$$

and express for convenience the function $\mathbf{v}(\lambda)$ in the parametric form

$$
\begin{aligned}
& \mathbf{v}=\mathbf{v}(t) \\
& \lambda=\lambda(t)
\end{aligned}
$$

where $t=0$ is assumed to correspond to bifurcation. We admit that the functions in eqn (4) can be represented in the neighbourhood of $t=0$ by the series expansions

$$
\begin{aligned}
& \lambda(t)=\lambda_{c}+\dot{\lambda}_{c} t+0\left(t^{2}\right) \\
& \mathbf{v}(t)=\mathbf{v}_{c} t+0\left(t^{2}\right)
\end{aligned}
$$

in which here and in the sequel the subscript $c$ refers to the bifurcation point and a dot denotes $t$-differentiation. Note that second order terms have been omitted in eqn (5) since we are only interested in the evaluation of the critical mode $\dot{v}_{c}$ and of the tangent $\dot{\lambda}_{c}$ to the bifurcated path. In 
problems in which $\dot{\lambda}_{c}=0$, the postbuckling path is symmetric, and second order terms are necessary for a meaningful analysis of the mechanical behaviour of the system. The first two coefficients of the series expansions in eqn (5), $\lambda_{c}, \dot{\mathbf{v}}_{c}$, are obtained by solving the eigenvalue problem

$$
\Phi_{c}^{\prime \prime}\left[\mathbf{u}_{c} ; \lambda_{c}\right] \dot{\mathbf{v}}_{c} \delta \mathbf{u}=0 \quad \forall \delta \mathbf{u}
$$

Equation (6) is obtained by taking the first $t$-derivative of eqn (1) along the fundamental and bifurcated paths and by subtracting by correspondence with $t=0$.

It may happen that the eigenvalue problem in eqn (6) admits a multiple eigenvalue $\lambda_{c}$ of multiplicity $m$. The most general solution of eqn (6) can then be expressed as a linear combination of $m$ eigenvectors associated with $\lambda_{c}$, that is

$$
\dot{v}_{c}=\nu_{i} \dot{\mathbf{v}}_{i c}
$$

where repeated indices denote summation from 1 to $m$ and $\nu_{i}$ are parameters satisfying

$$
\nu_{i} \nu_{i}=1
$$

which describe the relative contributions of the various modes to the additional displacement $\mathbf{v}$.

To determine $\dot{\lambda}_{c}$ we need to make use of the equation relative to the evaluation of the second order term $\ddot{\mathbf{v}}_{c}$ of the series expansion (5)

$$
\begin{aligned}
\Phi_{c}^{\prime \prime} \ddot{\mathbf{v}}_{c} \delta \mathbf{u}=-\left\{\Phi_{c}^{\prime \prime \prime}\left(\nu_{i} \dot{\mathbf{v}}_{i c}\right)^{2}\right. & +2 \dot{\lambda}_{c} \Phi_{c}^{\prime \prime \prime} \hat{\mathbf{u}}_{c}^{\mathrm{f}}\left(\nu_{i} \dot{\mathbf{v}}_{i c}\right)+ \\
& \left.+2 \dot{\lambda}_{c} \hat{\Phi}_{c}^{\prime \prime}\left(\nu_{i} \dot{\mathbf{v}}_{i c}\right)\right\} \delta \mathbf{u} \quad \forall \delta \mathbf{u}(i=1, \ldots, m)
\end{aligned}
$$

where a hat denotes differentiation with respect to $\lambda$ and the arguments of the functionals $\Phi_{c}^{\prime \prime}, \Phi_{c}^{\prime \prime \prime}, \hat{\Phi}_{c}^{\prime \prime}$ have been omitted for simplicity. Equation (9) is obtained along the same lines as eqn (6) starting from the second $t$-derivative of eqn (1). Due to the singularity of the self-adjoint operator $\Phi_{c}^{\prime \prime}$, eqn (9) has a solution if and only if its right-hand member is orthogonal to the solutions of the homogeneous problem, eqn (6). Hence by assuming $\delta \mathbf{u}=\dot{\mathbf{v}}_{i c}, \ldots, \dot{\mathbf{v}}_{m c}$, successively, eqn (9) in conjunction with eqn (6) yields a set of $m$ equations of the type

$$
\Phi_{c}^{\prime \prime \prime}\left(\nu_{i} v_{i c}\right)^{2} \boldsymbol{v}_{j c}+2 \dot{\lambda}_{c} \Phi_{c}^{\prime \prime \prime} \hat{\mathbf{u}}_{c}^{\mathrm{f}}\left(\nu_{i} \boldsymbol{v}_{i c}\right) \hat{\mathbf{v}}_{j c}+2 \dot{\lambda}_{c} \hat{\Phi}_{c}^{\prime \prime}\left(\nu_{i} \dot{\boldsymbol{v}}_{i c}\right) \dot{\mathbf{v}}_{j c}=0 \quad(j=1, \ldots, m)
$$


which together with the conditions in eqn (8) permit evaluation of the $m$ numbers $\nu_{i}$ and $\dot{\lambda}_{c}$. Since eqn (10) is nonlinear, according to Bézout's theorem ${ }^{20}$ there are at most $2^{m}-1$ essentially different solutions and at least one real solution.

For a single buckling mode eqn (10) furnishes

$$
\dot{\lambda}_{c}=-\frac{1}{2} \frac{\Phi_{c}^{\prime \prime \prime} \dot{\boldsymbol{v}}_{c}^{3}}{\boldsymbol{\Phi}_{c}^{\prime \prime \prime} \hat{\mathbf{u}}_{c}^{\mathbf{v}} \mathbf{v}_{c}^{2}+\hat{\Phi}_{c}^{\prime \prime} \mathbf{v}_{c}^{2}}
$$

\section{KINEMATICS AND POTENTIAL ENERGY FORMULATION}

Let us consider a channel under a system of uniformly distributed axial forces at the ends and further assume that the end constraint allows only axial displacements (simply supported column). Field equations will be derived consistent with the assumption that the column is a plate assemblage with straight junction lines.

On the basis of a purely linear kinematics, the displacements field is described following the classical Vlasov theory ${ }^{21}$ where the assumption of the rigid cross-section is removed. In the sequel it will be convenient to express the (linearized) total points displacement as the sum of the Vlasov (global) displacement and an additional one (local) in which the crosssection is assumed to lose its shape the junction lines position remaining unchanged.

In order to obtain a representation for the global (linearized) displacement field it is useful to make the following decomposition

$$
\mathbf{u}^{\mathrm{g}}(P)=\mathbf{u}_{\alpha}^{\mathrm{g}}(P)+\mathbf{u}_{\pi}^{\mathrm{g}}(P)
$$

where $\mathbf{u}_{\alpha}^{\mathrm{g}}(P)$ and $\mathbf{u}_{\pi}^{\mathrm{g}}(P)$ are the orthogonal projections of $\mathbf{u}^{\mathrm{g}}(P)$ onto the beam axis and the cross-section plane, respectively. Following Vlasov we assume $\mathbf{u}_{\pi}^{\mathrm{g}}(P)$ to be rigid in the sense that

$$
\mathbf{u}_{\pi}^{\mathrm{g}}(P)=\mathbf{u}_{\pi}^{\mathrm{g}}(Q)+\boldsymbol{\theta} \times(P-Q)
$$

for all $P, Q$ belonging to the same cross-section plane, $\theta$ being the rotation vector.

Let us consider now the channel in its undeformed shape and introduce an orthonormal basis $\left(\mathbf{e}_{1}, \mathbf{e}_{2}, \mathbf{e}_{3}\right)$ such that $\mathbf{e}_{1}$ is in the direction of the beam 


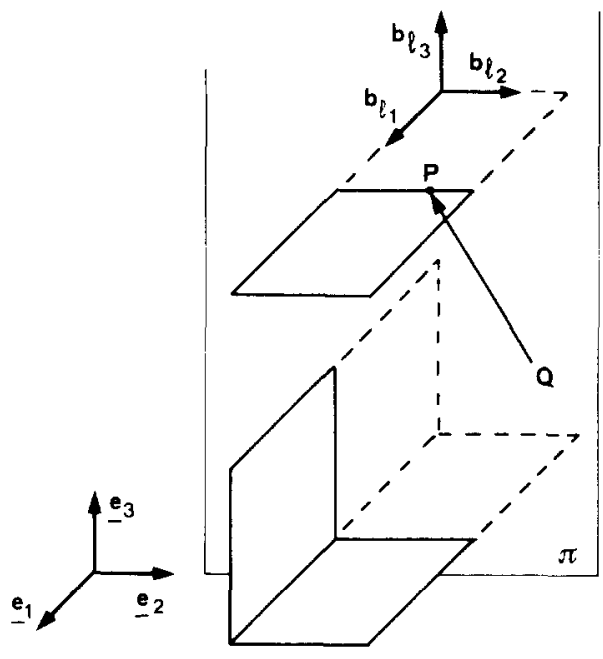

a)

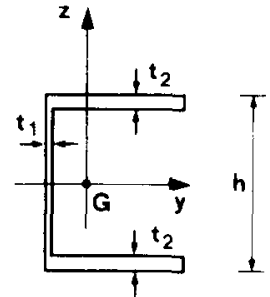

b

b)

Fig. 1. (a) Local coordinate system; (b) cross-section geometry.

axis and $\mathbf{e}_{2}, \mathbf{e}_{3}$ are parallel to the cross-section central axes of inertia $y$ and $z$, respectively (Fig. 1).

For each plate of the folded structure we introduce now a (local) coordinate system $x, s_{l}$ with origin $O_{l}$ and natural (orthonormal) basis $\left(\mathbf{b}_{11} \equiv \mathbf{e}_{1}, \mathbf{b}_{12}\right)$. A (local) rectangular Cartesian coordinate system $x, s_{l}, n_{l}$ covering the whole space can then be obtained by introducing the basis $\left(\mathbf{e}_{1}, \mathbf{b}_{12}, \mathbf{b}_{13} \equiv \mathbf{e}_{1} \times \mathbf{b}_{l 2}\right.$ ) (Fig. 1a). Components of the displacement field are now defined through

$$
\mathbf{u}^{\mathrm{g}}(P)=u^{\mathrm{g}}(P) \mathbf{e}_{1}+v^{\mathrm{g}}(P) \mathbf{b}_{l 2}+w^{\mathrm{g}}(P) \mathbf{b}_{l 3}
$$

where $u^{\mathrm{g}}(P)=\mathbf{u}_{\alpha}^{\mathrm{g}}(P) \cdot \mathbf{e}_{1}, \quad v^{\mathrm{g}}(P)=\mathbf{u}_{\pi}^{\mathrm{g}}(P) \cdot \mathbf{b}_{l 2}, \quad w^{\mathrm{g}}(P)=\mathbf{u}_{\pi}^{\mathrm{g}}(P) \cdot \mathbf{b}_{l 3}$ and $P=P\left(x, s_{l}\right)$. By enforcing the condition (Vlasov)

$$
\gamma=\frac{\partial u^{g}}{\partial s_{l}}+\frac{\partial v^{g}}{\partial x}=0
$$


we obtain

$$
u^{\mathrm{g}}\left(s_{l}\right)=\bar{u}_{l}-\int_{0_{l}}^{s_{l}}\left[\frac{\partial \mathbf{u}_{\pi}^{\mathrm{g}}(Q)}{\partial x} \cdot \mathbf{b}_{l 2}\right] \mathrm{d} s_{l}-\frac{\partial \boldsymbol{\theta}}{\partial x} \cdot \omega\left(Q, O_{l}, s_{l}\right)
$$

where

$$
\boldsymbol{\omega}\left(Q, O_{l}, s_{l}\right)=\int_{0_{l}}^{s_{l}}\left[P\left(s_{l}\right)-Q\right] \times \mathbf{b}_{l 2} \mathrm{~d} s_{l}
$$

is the sectorial area with pole $Q$ and zero point $O_{l}$ and the dependence of $u^{\mathrm{g}}, \bar{u}, Q, \theta$ on $x$ has been omitted. With reference to a generic crosssection, it can now be easily seen that

$$
\begin{aligned}
& \mathbf{b}_{l 2}=\frac{\partial}{\partial s_{l}}\left(P\left(s_{l}\right)-G\right) \\
& \omega\left(Q, O_{l}, s_{l}\right)=\omega\left(Q, M, s_{l}\right)+\mathbf{k}\left(Q, O_{l}, M\right)
\end{aligned}
$$

where $G$ is the centroid of the cross-section, $M$ the zero point for $\omega\left(Q, M, s_{l}\right)$ which coincides with the principal zero point of the crosssection and $\mathbf{k}\left(Q, O_{l}, M\right)$ is a vector independent of $s_{l}$. If we let $Q$ coincide with the shear centre $S$, eqns (16) and (13) can be written in the form

$$
\begin{aligned}
& u^{\mathrm{g}}\left(s_{l}\right)=\tilde{u}-\frac{\partial \mathbf{u}_{\pi}(S)}{\partial x} \cdot\left[P\left(s_{l}\right)-G\right]-\frac{\partial \boldsymbol{\theta}}{\partial x} \cdot \boldsymbol{\omega}\left(S, M, s_{l}\right) \\
& \mathbf{u}_{\pi}^{\mathrm{g}}\left(s_{l}\right)=\mathbf{u}_{\pi}(S)+\boldsymbol{\theta} \times\left[P\left(s_{l}\right)-S\right]
\end{aligned}
$$

$\tilde{u}$ being an arbitrary function of $x$. In order to ensure compatibility for $u$ along the junction lines $\tilde{u}$ must be the same for all plates. In particular we will assume $\tilde{u} \equiv 0$.

By defining the following components

$$
\begin{aligned}
& \mathbf{u}_{\pi}(S)=v_{S}(x) \mathbf{e}_{2}+w_{s}(x) \mathbf{e}_{3} \\
& \boldsymbol{\theta}=\boldsymbol{\theta}(x) \mathbf{e}_{1} \\
& \boldsymbol{\omega}\left(S, M, s_{l}\right)=\omega\left(s_{l}\right) \mathbf{e}_{1} \\
& P\left(s_{l}\right)-S=c_{2}\left(s_{l}\right) \mathbf{e}_{2}+c_{3}\left(s_{l}\right) \mathbf{e}_{3} \\
& P\left(s_{l}\right)-G=g_{2}\left(s_{l}\right) \mathbf{e}_{2}+g_{3}\left(s_{l}\right) \mathbf{e}_{3}
\end{aligned}
$$


the scalar counterpart of eqn (19) reads

$$
\begin{aligned}
& u^{\mathrm{g}}\left(x, s_{l}\right)=-v_{s}^{\prime}(x) g_{2}\left(s_{l}\right)-w_{s}^{\prime} g_{3}\left(s_{l}\right)-\theta^{\prime}(x) \omega\left(s_{l}\right) \\
& v^{\mathrm{g}}\left(x, s_{l}\right)=v_{s}(x)-\theta(x) c_{3}\left(s_{l}\right) \\
& w^{\mathrm{g}}\left(x, s_{l}\right)=w_{s}(x)+\theta(x) c_{2}\left(s_{l}\right)
\end{aligned}
$$

where a prime from now on will denote differentiation with respect to $x$.

The local (linearized) displacement field is described consistently with the assumption

$$
\mathbf{u}^{l}(P) \equiv \mathbf{u}_{\pi}^{l}(P)=w^{l}(P) \mathbf{b}_{l 3}
$$

It is useful to stress that the assumption in eqn (22) implies $\mathbf{u}^{\prime}(P) \equiv \mathbf{0}$ all along the lines in which the web joins the flanges. The effective (linearized) displacement field is then obtained by combining eqns (12) and (22)

$$
\begin{aligned}
\mathbf{u}(P) & =\mathbf{u}^{\mathrm{g}}(P)+\mathbf{u}^{\prime}(P) \\
& =u(P) \mathbf{e}_{1}+v(P) \mathbf{b}_{l 2}+w(P) \mathbf{b}_{l 3}
\end{aligned}
$$

Due to eqn (22) it is immediately seen that

$$
\begin{aligned}
& u(P) \equiv u^{\mathrm{g}}(P) \\
& v(P) \equiv v^{\mathrm{g}}(P) \\
& w(P)=w^{\mathrm{g}}(P)+w^{l}(P)
\end{aligned}
$$

Under the assumption that the channel is made of a hyperelastic, homogeneous material, we introduce the total potential energy function in the form

$$
\Phi=\sum_{l}^{3} \int_{A_{l}} \varphi_{l} \mathrm{~d} A_{l}-\int_{\mathscr{E}_{l}} \lambda N_{x x}\left[u\left(o, s_{l}\right)-u\left(l, s_{l}\right)\right] \mathrm{d} s_{l}
$$

where $A_{l}$ is the plate surface, $\mathscr{C}_{l}$ the end contour on which the external load $\lambda N_{x x}$ is applied and $\varphi_{l}$ is the strain energy density of each plate. We assume $\varphi_{l}$ to be represented through a quadratic expression in terms of the in-plane and curvature strain measures $\epsilon$ and $\chi$, respectively, without coupling terms

$$
\begin{aligned}
& \varphi_{l}(\epsilon, \chi)=\frac{1}{2}\left\{D_{\mathrm{a}}\left[\left(\epsilon_{x x}+\epsilon_{s s}\right)^{2}-2(1-\nu)\left(\epsilon_{x x} \epsilon_{s s}-\epsilon_{x s}^{2}\right)\right]\right. \\
& \left.+D_{f}\left[\left(\chi_{x x}+\chi_{s s}\right)^{2}-2(1-\nu)\left(\chi_{x x} \chi_{s s}-\chi_{x s}^{2}\right)\right]\right\}
\end{aligned}
$$


Here $D_{a}=E t /\left(1-\nu^{2}\right), D_{f}=E t^{3} / 12\left(1-\nu^{2}\right)$ are the axial and bending stiffnesses, respectively, $\nu$ is the Poisson ratio, $E$ the Young modulus while $\epsilon$ and $\chi$ are the components of the strain measures.

The strain-displacement relationships are listed hereafter

$$
\begin{aligned}
& \boldsymbol{\epsilon}_{x x}=u^{\prime}+\frac{1}{2}\left[\left(u^{\prime}\right)^{2}+\left(v^{\prime}\right)^{2}+\left(w^{\prime}\right)^{2}\right] \\
& \boldsymbol{\epsilon}_{s s}=-\boldsymbol{\nu} \boldsymbol{\epsilon}_{x x} \\
& \boldsymbol{\epsilon}_{x s}=\frac{1}{2}\left(u^{\prime} u^{\prime}+w^{\prime} w^{\prime}\right) \\
& \chi_{x x}=\left(w^{\prime}\right)^{2} \\
& \chi_{s s}=\left(w^{\prime}\right)^{2} \\
& \chi_{x s}=w^{\prime \prime}
\end{aligned}
$$

where the apostrophe, from now on, will denote differentiation with respect to $s_{l}$. By replacing the expressions from eqn (27) into eqn (26) we obtain, up to third order terms

$$
\begin{aligned}
\varphi_{l}=\frac{1}{2}\{ & E t\left[\left(u^{\prime}\right)^{2}+u^{\prime}\left(\left(u^{\prime}\right)^{2}+\left(v^{\prime}\right)^{2}+\left(w^{\prime}\right)^{2}\right)\right] \\
& \left.+D_{f}\left[\left(w^{\prime \prime}\right)^{2}+\left(w^{\prime \prime}\right)^{2}+2 \nu w^{\prime \prime} w^{\prime \prime}+2(1-\nu)\left(w^{\prime \prime}\right)^{2}\right]\right\}
\end{aligned}
$$

In view of obtaining the counterpart of eqns (6) and (10), respectively. for the problem at hand, we need the second and third variation of eqn (28) which read

$$
\begin{aligned}
\varphi_{1}^{\prime \prime} u_{1} u_{2}=E t & {\left[u_{1}^{\prime} u_{2}^{\prime}+3 u^{\prime} u_{1}^{\prime} u_{2}^{\prime}+u^{\prime}\left(v_{1}^{\prime} v_{2}^{\prime}+w_{1}^{\prime} w_{2}^{\prime}\right)\right.} \\
& \left.+v^{\prime}\left(u_{1}^{\prime} v_{2}^{\prime}+v_{1}^{\prime} u_{2}^{\prime}\right)+w^{\prime}\left(u_{1}^{\prime} w_{2}^{\prime}+w_{1}^{\prime} u_{2}^{\prime}\right)\right] \\
& +D_{f}\left[w_{1}^{\prime} w_{2}^{\prime}+w_{1}^{\prime} w_{2}^{\prime}+\nu\left(w_{1}^{\prime} w_{2}^{\prime \prime}+w_{1}^{\prime \prime} w_{2}^{\prime \prime}\right)+2(1-\nu) w_{1}^{\prime} w_{2}^{\prime}\right] \\
\varphi_{l}^{\prime \prime} u_{1} u_{2} u_{3}= & E t\left[3 u_{1}^{\prime} u_{2}^{\prime} u_{3}^{\prime}+u_{1}^{\prime}\left(v_{2}^{\prime} v_{3}^{\prime}+w_{2}^{\prime} w_{3}^{\prime}\right)+v_{1}^{\prime}\left(u_{2}^{\prime} v_{3}^{\prime}+v_{2}^{\prime} u_{3}^{\prime}\right)\right. \\
& +w_{1}^{\prime}\left(u_{2}^{\prime} w_{3}^{\prime}+w_{2}^{\prime} u_{3}^{\prime}\right)
\end{aligned}
$$

It is easily verified that the fundamental path in our case is

$$
\begin{gathered}
u^{\mathrm{f}}(x)=-\frac{\lambda N_{x x}}{E t} x \\
v^{\mathrm{f}}(x) \equiv w^{\mathrm{f}}(x) \equiv 0
\end{gathered}
$$

so that, evaluating eqn (29) along it leads to

$$
\begin{aligned}
\varphi_{l}^{\prime \prime}\left(u^{\mathrm{f}}\right) u_{1} u_{2}=E t & {\left[u_{1}^{\prime} u_{2}^{\prime}-\frac{\lambda N_{x x}}{E t}\left(3 u_{1}^{\prime} u_{2}^{\prime}+v_{1}^{\prime} v_{2}^{\prime}+w_{1}^{\prime} w_{2}^{\prime}\right)\right] } \\
& +D_{f}\left[w_{1}^{\prime} w_{2}^{\prime}+w_{1}^{\prime} w_{2}^{\prime}+\nu\left(w_{1}^{\prime \prime} w_{2}^{\prime \prime}+w_{1}^{\prime \prime} w_{2}^{\prime \prime}\right)\right. \\
& \left.+2(1+\nu) w_{1}^{\prime \prime} w_{2}^{\prime \prime}\right]
\end{aligned}
$$




\section{DISCRETE ANALYSIS}

The problem presented in the previous sections can be given a discrete formulation suitable for numerical analysis. Starting from eqn (21) we assume

$$
\begin{aligned}
& v_{S}(x)=V_{S} \sin \pi x / l \\
& w_{\mathrm{S}}(x)=W_{S} \sin \pi x / l \\
& \theta(x)=\theta \sin \pi x / l
\end{aligned}
$$

which satisfy the simply supported boundary conditions and allow a description of the global displacement field in terms of the parameters $V_{s}, W_{s}, \theta$.

Passing now to the local displacement field we shall assume

$$
w^{l}\left(x, s_{\ell}\right)=\mathrm{f}(x) g\left(s_{l}\right)
$$

where $\mathrm{f}(x)$ is a sine and $g\left(s_{l}\right)$ is a polynomial function. Further we divide the channel into four strips (Fig. 2) and assume for each strip the displacement field to be expressed in the form

$$
w^{l}\left(x, s_{i}\right)=\mathrm{f}(x) g\left(s_{i}\right)
$$

where $\mathrm{f}(x)$ is a sinusoidal function representing displacements and rotations of the nodal lines, and $g\left(s_{i}\right)$ are cubic polynomials. The functions $\mathrm{f}(x)$ employed in the analysis are listed below

$$
\begin{aligned}
w_{A}(x) & =W_{A} \sin n \pi x / l \\
\theta_{B}(x) & =\theta_{B} \sin n \pi x / l \\
w_{H}(x) & =W_{H} \sin n \pi x / l \\
\theta_{H}(x) & =\theta_{H} \sin n \pi x / l \\
\theta_{C}(x) & =\Theta_{C} \sin n \pi x / l \\
w_{D}(x) & =W_{D} \sin n \pi x / l
\end{aligned}
$$

where $n$ is an integer and $W_{A}, \theta_{B}, W_{H}, \theta_{H}, \theta_{C}, W_{D}$ are the parameters assumed to describe the configuration. Note that the rotations in $A$ and $D$ have been condensed by requiring $w_{A}^{\prime \prime}(x) \equiv w_{D}^{\prime}(x) \equiv 0$.

We can now obtain, for each element, a discrete counterpart of eqn (32) by substituting for $u_{1}, v_{1}, w_{1}$ and $u_{2}, v_{2}, w_{2}$ the discrete expressions. 


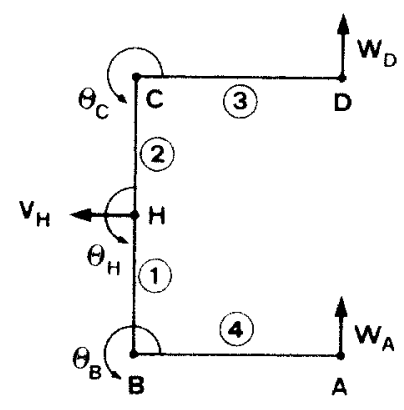

Fig. 2. Degrees of freedom of the discretized channel.

After the double integration along $x$ and $s_{l}$ has been performed, we arrive at a system of equations that define a linear algebraic eigenvalue problem in terms of $\lambda$.

The steps aforementioned have been performed by means of the automatic Algebraic Manipulation System (AMS) REDUCE, running on the UNIVAC 1100 at the computer centre of the University of Rome. ${ }^{22-24}$

Due to the few strips employed and to the problem symmetries, the use of the AMS with its possibilities to define integration procedures, has allowed solution of our problem practically in parametric form. Numerical computations have been performed only in solving the eigenvalue problem.

In the same fashion second and third order terms necessary to build up the discrete counterpart of eqn (10) have been easily obtained by means of the AMS.

It is worth noting that the procedure described herein can be utilized as a support for a finite element of finite strip analysis. ${ }^{25}$ For a discussion on the use of the AMS with mixed perturbation/Galerkin techniques, see also ref. 26.

\section{BUCKLING ANALYSIS}

An extensive parametric analysis has been performed by using a FORTRAN program generated by the program of algebraic manipulation. The local and overall buckling modes and the associated critical stresses have been determined for different geometries of beams of technical interest. In particular, with regard to overall buckling modes, it 
is found that the critical stress is affected by the web/flange thickness ratio due to the loss of shape of the cross-section. However, since our analysis is focused on the behaviour of cold formed steel members (constant thickness), this effect is practically irrelevant and Vlasov theory can be successfully employed. Thus we have

$$
\begin{aligned}
\sigma_{F T} & =\frac{1}{2 c}\left[\left(\sigma_{y}+\sigma_{\theta}\right)-\sqrt{\left.\left(\sigma_{y}+\sigma_{\theta}\right)^{2}-4 c \sigma_{y} \sigma_{\theta}\right]}\right. \\
\sigma_{E} & =\sigma_{z}
\end{aligned}
$$

Here $\sigma_{y}, \sigma_{z}$ and $\sigma_{\theta}$ are the well known flexural and torsional buckling stresses, respectively, and $c=1-\left(y_{s} / r_{s}\right)^{2}$, where $y_{s}$ is the abscissa of the shear centre in the principal coordinate frame and $r_{s}$ is the radius of gyration with respect to the same point.

By introducing the nondimensional parameters

$$
\alpha=l / h \quad \beta=b / h \quad \gamma=t / h
$$

where $l$ is the beam length and $b, h, t$ are defined in Fig. $1 \mathrm{~b}$, a number of diagrams corresponding to different classes of beams have been plotted. Figures 3 to 5 furnish in a semi-log scale the values of the critical stress $\sigma_{c} / E v s \alpha$ for fixed values of $\beta$, and $\gamma$ ranging approximately between $10^{-3}$ and $10^{-1}$. It is seen that, whereas the purely flexural buckling curve is independent of $\gamma$, the flexural-torsional curves are affected by this parameter and cover the lower area of the diagrams for decreasing values of $\gamma$. Besides, for decreasing values of $\beta$ the lowest flexural-torsional curve approaches the eulerian curve, and for $\beta$ sufficiently small and $\gamma$ not exceeding the prescribed range, the buckling mode is only purely flexural. Points of intersection of the two families of curves correspond to simultaneous overall buckling modes.

Local buckling analysis has been performed numerically by using the discrete plate assembly model. Results have been tested for a number of limit cases corresponding to values of the flange/web thickness ratio around zero and infinity, thus obtaining the critical stress of simply supported or longitudinally clamped plate. For constant thickness, results furnished by ref. 27 have been recovered and plotted in Fig. 6. It is found that the local buckling mode of the cross-section is symmetric with respect to the $y$-axis and it is represented in Fig. 6 for two different values of $\beta$. 


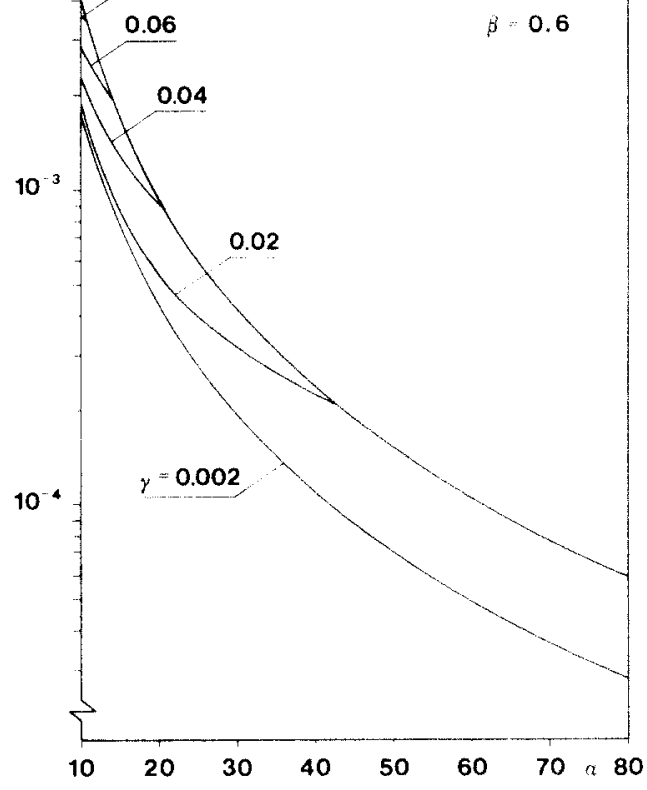

Fig. 3. Overall buckling stress $v s \alpha$.

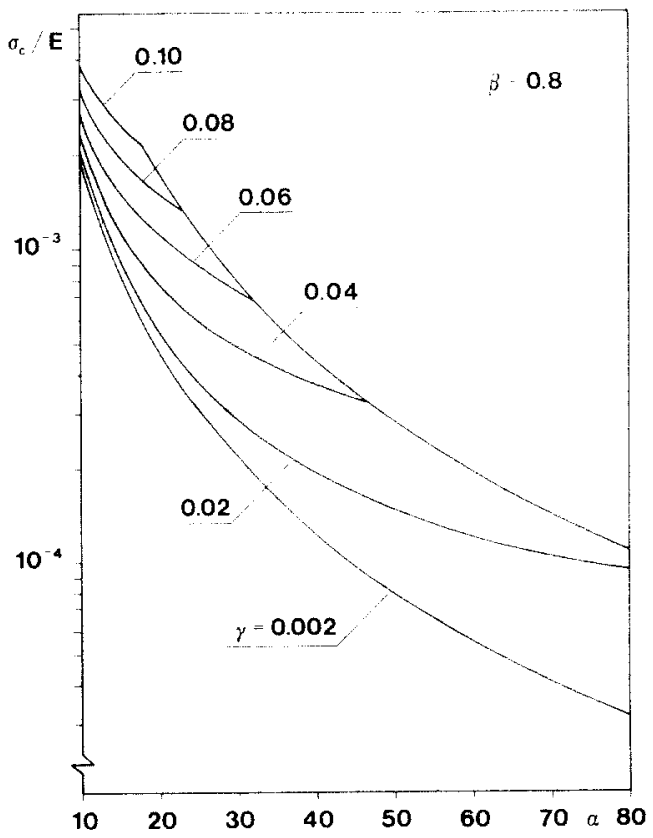

Fig. 4. Overall buckling stress vs $\alpha$. 


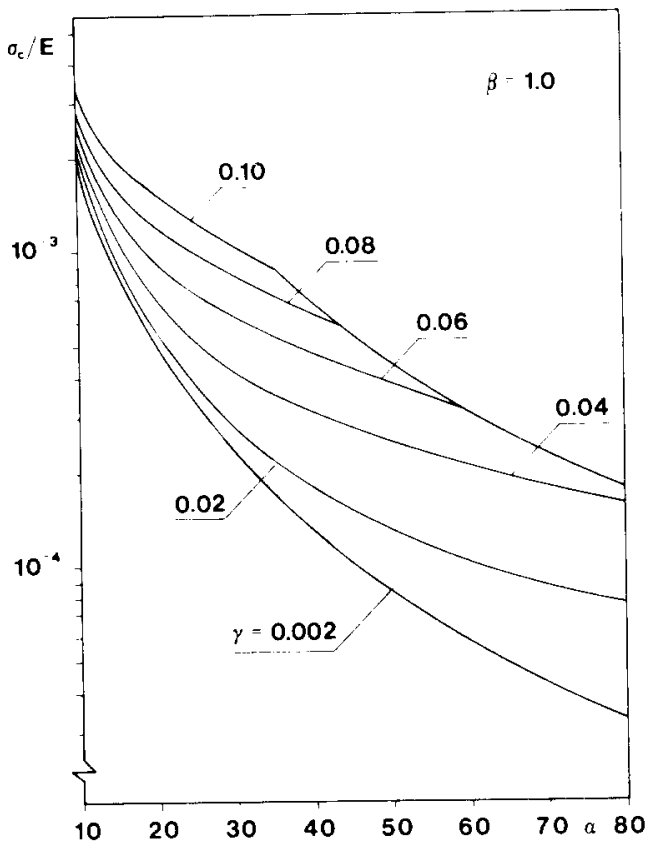

Fig. 5. Overall buckling stress vs $\alpha$.

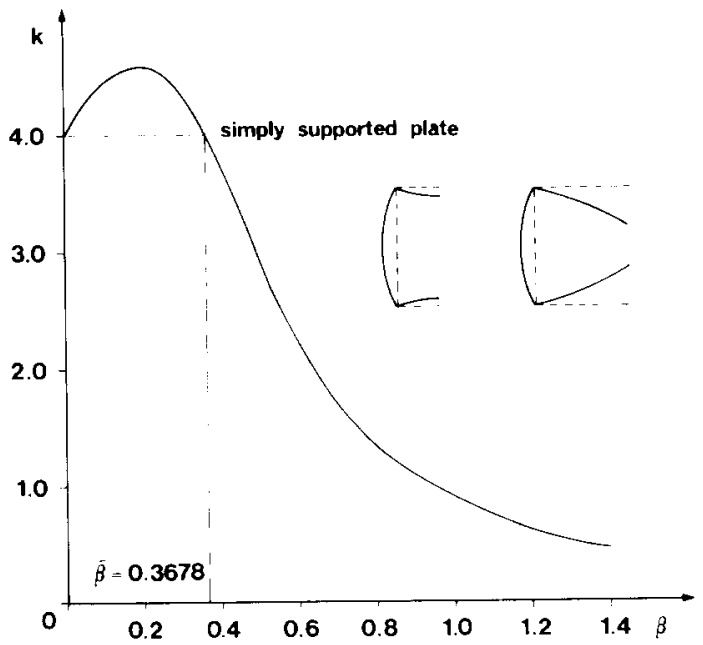

Fig. 6. Coefficient $k$ in eqn (39) vs $\beta$. 
The critical stress $\sigma_{L} / E$ is length-independent for not too short beams and it is proportional to $\gamma^{2}$ according to

$$
\sigma_{L}=k \frac{\pi^{2}}{12} \frac{E}{1-\nu^{2}} \gamma^{2}
$$

where $k(\beta)$ is furnished by the diagram for $\nu=0 \cdot 3$.

It is observed that for $\beta$ approaching zero the value $k=4$ corresponding to simply supported plate is obtained. A small increment of $\beta$ up to about $0 \cdot 2$ increases the structure stiffness and consequently the critical stress. In this case the thin-walled member behaviour is the same as that of a plate elastically supported along the longitudinal edges. For larger values of $\beta$ the stiffening effect due to the flanges tends to diminish and for $\beta=\bar{\beta}=0.3678$ the value $k=4$ is recovered. From a mechanical point of view this can be explained by thinking that the stiffening effects of the flanges are counterbalanced by the possibility that the buckling is initiated by the flanges themselves. For increasing values of $\beta$ the destabilizing effect obviously prevails and therefore $k$ decreases rapidly.

We look now for the conditions under which simultaneous buckling modes (overall/overall and local/overall) occur. By equating two by two the right hand members of eqns (37) and (39) we obtain the simple relations

$$
\alpha \gamma=\phi_{i}(\beta) \quad(i=1,2,3)
$$

The three functions $\phi_{t}(\beta)$ are explicitly given in the Appendix and are plotted in Fig. 7. For each value of $\beta$ we may select infinite pairs of values of $\alpha$ and $\gamma$ which satisfy eqn (40) such that two critical stresses coincide, their actual value depending on $\alpha$ and $\gamma$. From an engineering point of view it is interesting to determine in the space of the geometrical parameters the domains inside which single buckling modes occur and the boundaries of two adjacent domains along which two critical stresses coincide. After some simple manipulation it can be easily shown that the inequalities

$$
\sigma_{F T} \sigma_{E}, \quad \sigma_{L} \sigma_{E}, \quad \sigma_{L}
$$

are satisfied if the geometrical parameters verify the relations

$$
\alpha \gamma \phi_{1}(\beta), \quad \alpha \gamma \phi_{2}(\beta), \quad \alpha \gamma \phi_{3}(\beta)
$$




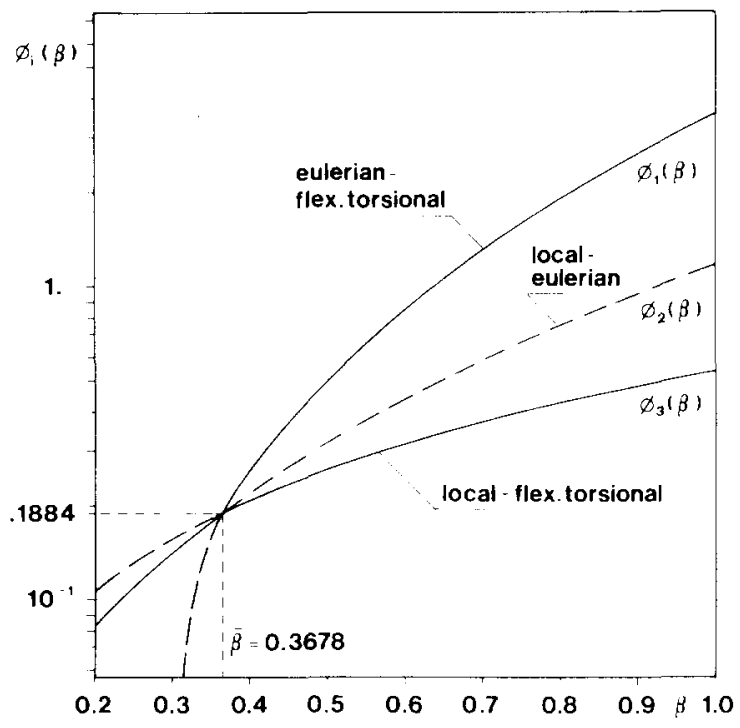

Fig. 7. Functions $\phi_{i}$ in eqn (40) vs $\beta$.

The inequalities in eqn (42) can be usefully employed to verify whether or not the critical stress corresponding to two interacting modes is the smallest possible in the column. If we consider, for instance, the interaction eulerian/flexural-torsional, the corresponding curve $\phi_{1}(\beta)$ is meaningful only if $\sigma_{F T}=\sigma_{E}<\sigma_{L}$, i.e. by using eqns (41) and (42), for $\phi_{1}(\beta)>\phi_{2}(\beta)$ which is verified for $\beta>\bar{\beta}=0.3678$. For $\beta<\bar{\beta}$ the situation is reversed and the local critical stress prevails, the eulerian/ flexural-torsional buckling interaction occurring at a higher value of the loading. In a completely similar manner it can be shown that $\sigma_{E}=$ $\sigma_{L}<\sigma_{F T}$ if $\phi_{2}(\beta)<\phi_{3}(\beta)$ which takes place for $\beta<\bar{\beta}$ and besides $\sigma_{F T}=\sigma_{L}<\sigma_{E}$ if $\phi_{3}(\beta)<\phi_{2}(\beta)$ which occurs for $\beta>\bar{\beta}$. In conclusion, for $\beta>\bar{\beta}$ two different types of interaction can manifest, namely the eulerian/flexural-torsional or the local/flexural-torsional; for $\beta<\bar{\beta}$ only the local/eulerian interaction is possible. For $\beta=\bar{\beta}$ the three buckling modes occur simultaneously. Note that curve $\phi_{1}(\beta)$ is not defined for values of $\beta<\beta^{*}=0.303$ and therefore the eulerian/flexural-torsional interaction can never exist below this value. In Fig. 7 the meaningful branches of the curves have been represented with heavy lines; dashed branches correspond to cases of no practical interest since there is always 

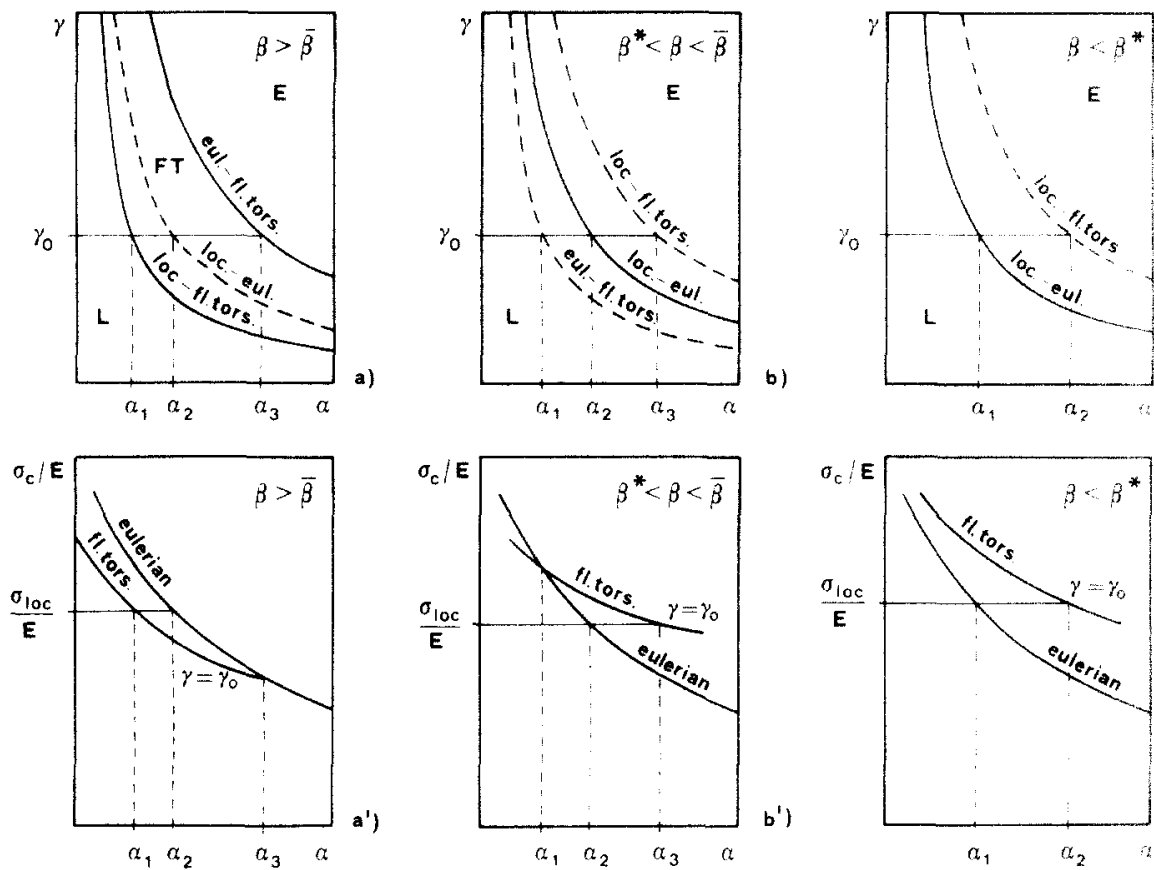

Fig. 8. Local/overall and overall/overall interaction buckling curves

a critical stress lower than that corresponding to the associated simultaneous buckling modes.

The previous arguments can be better illustrated by the diagrams in Fig. 8. In Fig. 8a, b and $\mathrm{c}$ hyperbolas defined by eqn (40) have been plotted for $\beta>\bar{\beta}, \beta^{*}<\beta<\bar{\beta}, \beta<\beta^{*}$. These curves delimit the domains in the $\alpha-\gamma$ plane inside which a single buckling mode occurs. With reference to Fig. 8a, for instance, the upper right region of the diagram corresponds to $\alpha \gamma>\phi_{1}(\beta)$ and therefore from eqn (41) $\sigma_{1} \sigma_{E}<\sigma_{F T}$ follows, this implying that the Euler buckling load prevails. Similar arguments hold true for the other domains. Below the previous figures, three qualitative diagrams of the type shown in Figs 3-5 have been sketched, corresponding to the same values of $\beta$. In each of them the eulerian buckling curve and one of the flexural-torsional buckling curves corresponding to a particular value of $\gamma=\gamma_{0}$ have been depicted. The associated local critical stress is also shown. Focusing our attention on Fig. 8a we can read three values of $\alpha$, related to $\gamma_{0}$ to which the three types of interaction are 
associated. Fig. $8 \mathbf{a}^{\prime}$ furnishes the corresponding critical stresses. It is apparent that the column related to $\alpha_{2}$ buckles at a load lower than $\sigma_{L}$ in a flexural-torsional mode. The same reasoning can be extended to the other two diagrams. Fig. $8 \mathrm{c}^{\prime}$ visualizes the previous statement that for $\beta<\beta^{*}$ the eulerian/flexural-torsional interaction does not occur.

\section{OVERALL/LOCAL INTERACTION POSTBUCKLING ANALYSIS}

Interaction buckling analysis is performed on the basis of the general theory presented in Section 2 where the investigation is restricted to the case of two interacting buckling modes, namely local/eulerian and local/ flexural-torsional. Eulerian/flexural-torsional interaction has also been tested, and results achieved in ref. 1 have been recovered.

By combining eqn (5) $)_{2}$ and (7) we have

$$
\mathbf{v}=\left(\nu_{1} \mathbf{v}_{1}+\nu_{2} \mathbf{v}_{2}\right) t+0\left(t^{2}\right)
$$

with the loading parameter expansion

$$
\frac{\lambda}{\lambda_{\mathrm{c}}}=1+\lambda_{1} t+0\left(t^{2}\right)
$$

where $\lambda_{1}=\dot{\lambda}_{c} / \lambda_{\mathrm{c}}$. On the other hand the counterpart of eqn (10) reads

$$
\begin{aligned}
& a_{11} \nu_{1}+a_{12} \nu_{1} \nu_{2}+a_{13} \nu_{2}^{2}+b_{11} \lambda_{1} \nu_{1}=0 \\
& a_{21} \nu_{1}^{2}+a_{22} \nu_{1} \nu_{2}+b_{22} \lambda_{1} \nu_{2}=0
\end{aligned}
$$

with the condition in eqn (8)

$$
\nu_{1}^{2}+\nu_{2}^{2}=1
$$

In eqn (45) and in the sequel indices 1 and 2 refer to overall and local buckling modes, respectively. The missing terms in eqn (45) turn out to be identically zero; besides coefficients $a_{12}, a_{21}$ vanish when the number $n$ of halfwaves in the local buckling mode is even. Explicit expression of the coefficients has been obtained by means of the AMS REDUCE as anticipated in Section 4. By solving eqns (45) and (46) three different solutions 
$\nu_{1}, \nu_{2}, \lambda_{1}$, at most, are found in accordance with Bézout theorem, and the corresponding bifurcated paths are determined through eqns (43) and (44). A direct relationship between the load parameter $\lambda$ and a meaningful displacement component of a particular point, say $u_{p}$, can be obtained by specializing eqn (43) to the evaluation of $u_{p}$ and replacing the corresponding value of $t$ into eqn (44). For both interaction analyses we find it convenient to select point $P$ coincident with a corner in such a way that no contribution to the displacement $\mathbf{v}$ arises from the local buckling mode. We have therefore

$$
\begin{aligned}
& \frac{\lambda}{\lambda_{\mathrm{c}}}=1+\bar{\lambda}_{1} \frac{\nu_{B}}{h} \\
& \frac{\lambda}{\lambda_{\mathrm{c}}}=1+\bar{\lambda}_{1} \frac{w_{B}}{h}
\end{aligned}
$$

for the local/eulerian and local/flexural interaction, respectively, where

$$
\bar{\lambda}_{1}=\frac{\lambda_{1} h}{\nu_{1} \dot{v}_{1 B}}, \quad \bar{\lambda}_{1}=\frac{\lambda_{1} h}{\nu_{1} \dot{w}_{1 B}}
$$

$v_{\mathrm{B}}$ and $w_{\mathrm{B}}$ being the global displacement component of corner $B$ at midspan as shown in Fig. 9.

From a kinematical point of view it can be interesting to evaluate the ratio between local and global displacement components along the

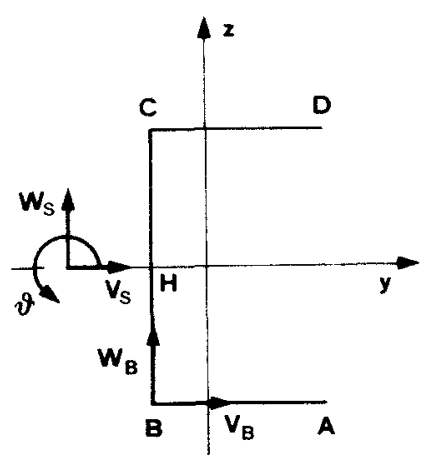

Fig. 9. Corners displacement components. 

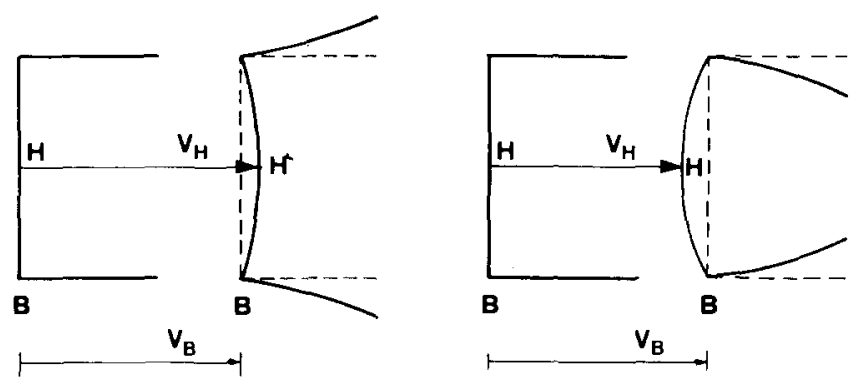

Fig. 10. Cross-section deformation in local/eulerian interaction.

bifurcated paths. As a meaningful displacement parameter for the local mode we select the wave amplitude at the point $H$, thus obtaining

$$
\frac{v_{H}-v_{B}}{v_{B}}=\frac{\nu_{2} \dot{v}_{2 H}}{\nu_{1} \dot{v}_{1 B}}
$$

for the local/eulerian interaction (Fig. 10) and

$$
\frac{v_{H}}{w_{B}}=\frac{\nu_{2} \dot{v}_{2 H}}{\nu_{1} \dot{w}_{1 B}}
$$

for the local/flexural-torsional interaction (Fig. 11).

With regard to the local/eulerian interaction, qualitative results can be achieved by analysing the nonlinear eqns (45) and (46). It is thus seen that
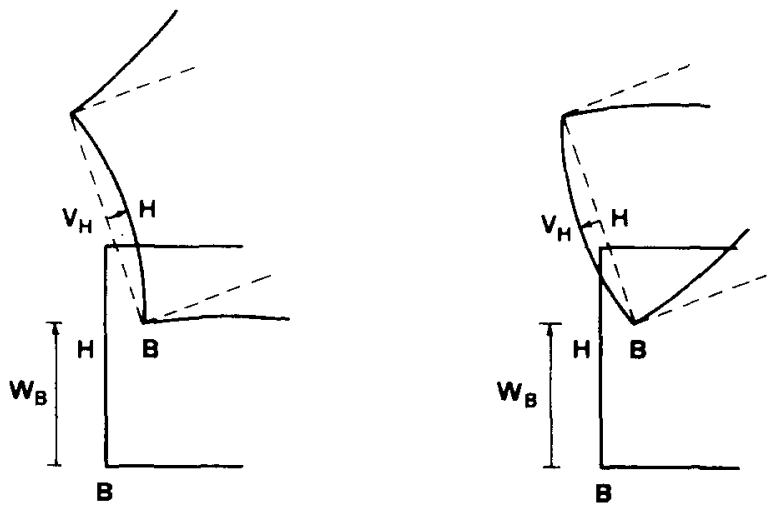

Fig. 11. Cross-section deformation in local/flexural-torsional interaction. 


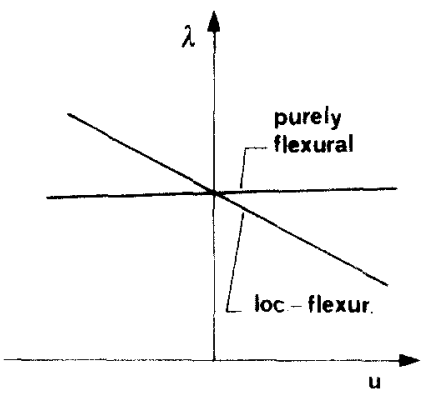

a)

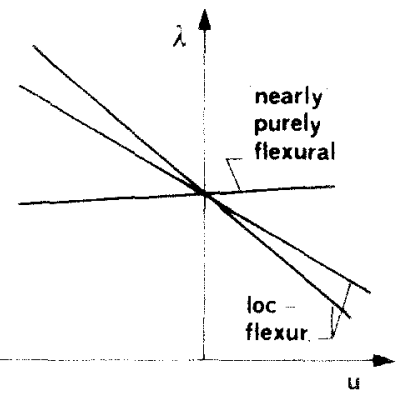

b)

Fig. 12. Local/eulerian postbuckling equilibrium paths: (a) $n$ even; (b) $n$ odd.

for $n$ even one solution is given by $\nu_{1}=1, \nu_{2}=0, \lambda_{1} \neq 0$ and corresponds to purely flexural buckling mode. Two other different solutions correspond to the triplets $\lambda_{1}, \nu_{1}, \nu_{2}$ and $\lambda_{1}, \nu_{1},-\nu_{2}$ (Fig. 10a and $b$, respectively) and are represented by the same straight line in Fig. 12a. This is obviously due to the fact that the configurations corresponding to the two solutions can be obtained from each other through a rigid rotation of the beam around the symmetry axis of midspan section. This is no longer true for $n$ odd and consequently the straight line is expected to split into two different ones (Fig. 12b). For very large values of $n$, however, the two situations become undistinguishable and therefore the two straight lines can be predicted to coincide again.

A number of beams corresponding to different values of the geometric parameters $\alpha, \beta, \gamma$ within the ranges defined in Section 5 have been analysed. Results are collected in Tables 1,2 and 3 corresponding to $\beta=0.20,0.25,0.30$, respectively, for different values of $\alpha$ and $\gamma$, such that $\alpha \gamma=\phi_{2}(\beta)$. It is apparent that for $n$ even the value $\bar{\lambda}_{1}$ corresponding to the purely flexural buckling mode is small but not zero, due to the presence of the term $u^{\prime 2}$ in the strain $\epsilon_{x x}$ definition, as has been checked from calculations. For $n$ odd there are three different solutions, one of which is 'nearly' purely flexural, as the value of the ratio in eqn (49) shows, and the other two correspond to slightly different values of $\bar{\lambda}_{1}$. For a fixed value of $x$, the associated cross-section deformations in eqn (49) along the two bifurcated paths are depicted in Fig. 10. For increasing values of $n$ it is apparent that these two solutions tend to coincide; besides for the three solutions the coefficient in eqn (49) approaches zero thus revealing a diminishing of the cross-section deformation. It is worth 


\section{TABLE 1}

Local/Eulerian Interaction $(\beta=0 \cdot 2)$

\begin{tabular}{|c|c|c|c|c|}
\hline$\alpha$ & $\gamma$ & $n$ & $\bar{\lambda}_{1}$ & $\left(v_{H}-v_{B}\right) / v_{B}$ \\
\hline $4 \cdot 21$ & 0.02 & 4 & $\left\{\begin{array}{r}-3.845 \\
0.068\end{array}\right.$ & $\begin{array}{c} \pm 0.593 \\
0\end{array}$ \\
\hline $8 \cdot 42$ & 0.01 & 9 & $\left\{\begin{array}{r}-4 \cdot 002 \\
-4 \cdot 164 \\
0 \cdot 022\end{array}\right.$ & $\begin{array}{r}0.271 \\
-0.255 \\
0 \cdot 005\end{array}$ \\
\hline $16 \cdot 84$ & $0 \cdot 005$ & 17 & $\left\{\begin{array}{r}-3.987 \\
-4.031 \\
0.005\end{array}\right.$ & $\begin{array}{r}0 \cdot 140 \\
-0 \cdot 137 \\
0.001\end{array}$ \\
\hline $41 \cdot 00$ & 0.002 & 41 & $\left\{\begin{array}{r}-3 \cdot 816 \\
-3 \cdot 823 \\
0.001\end{array}\right.$ & $\begin{array}{c}0.057 \\
-0.057 \\
1 \times 10^{-4}\end{array}$ \\
\hline
\end{tabular}

TABLE 2

Local/Eulerian Interaction $(\beta=0 \cdot 25)$

\begin{tabular}{|c|c|c|c|c|}
\hline$\alpha$ & $\gamma$ & $n$ & $\bar{\lambda}_{1}$ & $\left(v_{H}-v_{B}\right) / v_{B}$ \\
\hline $3 \cdot 67$ & 0.03 & 4 & $\left\{\begin{array}{r}-2 \cdot 552 \\
0 \cdot 105\end{array}\right.$ & $\begin{array}{c} \pm 0.609 \\
0\end{array}$ \\
\hline $5 \cdot 50$ & 0.02 & 5 & $\left\{\begin{array}{r}-1.977 \\
-2.448 \\
0.123\end{array}\right.$ & $\begin{array}{r}0.567 \\
-0.409 \\
0.049\end{array}$ \\
\hline $11 \cdot 00$ & 0.01 & 11 & $\left\{\begin{array}{r}-2 \cdot 370 \\
-2 \cdot 457 \\
0 \cdot 014\end{array}\right.$ & $\begin{array}{r}0.221 \\
-0.209 \\
0.004\end{array}$ \\
\hline $22 \cdot 00$ & 0.005 & 22 & $\left\{\begin{array}{r}-2 \cdot 416 \\
0 \cdot 003\end{array}\right.$ & $\begin{array}{c} \pm 0 \cdot 107 \\
0\end{array}$ \\
\hline
\end{tabular}

noting that $\bar{\lambda}_{1}$ decreases for increasing values of $\beta$ and that it changes very little with $\alpha$ and $\gamma$, when $\beta$ is kept constant.

Considering local/flexural-torsional buckling interaction, we have from the algebraic maniplation analysis AMS that coefficients $a_{11}, a_{13}, a_{23}$ are always zero for any value of $n$. Consequently, since for $n$ even also the 


\section{TABLE 3}

Local/Eulerian Interaction $(\beta=0 \cdot 3)$

\begin{tabular}{|c|c|c|c|c|}
\hline$\alpha$ & $\gamma$ & $n$ & $\bar{\lambda}_{1}$ & $\left(v_{H}-v_{B}\right) / v_{B}$ \\
\hline $4 \cdot 72$ & 0.03 & 5 & $\begin{array}{r}-0.822 \\
-1.180 \\
0.166\end{array}$ & $\begin{array}{r}0.669 \\
-0.381 \\
0.078\end{array}$ \\
\hline $7 \cdot 08$ & 0.02 & 7 & $\begin{array}{r}-0.710 \\
-0.881 \\
0.059\end{array}$ & $\begin{array}{r}0.402 \\
-0.289 \\
0.034\end{array}$ \\
\hline $14 \cdot 17$ & $0 \cdot 01$ & 14 & $\begin{array}{r}-0.781 \\
0.008\end{array}$ & $\begin{array}{c} \pm 0 \cdot 165 \\
0\end{array}$ \\
\hline $34 \cdot 56$ & 0.0041 & 33 & $\begin{array}{r}-0.727 \\
-0.735 \\
0.001\end{array}$ & $\begin{array}{r}0.070 \\
-0.069 \\
4 \times 10^{-}\end{array}$ \\
\hline
\end{tabular}

remaining coefficients $a_{12} a_{21}$ are zero, we have identically $\lambda_{1}=0$, for all bifurcated paths (Fig. 13a). The number of these curves cannot, however, be determined on the basis of the present analysis and higher order terms have to be considered. For $n$ odd eqn (45) reduces to

$$
\begin{aligned}
& a_{12} \nu_{1} \nu_{2}+b_{11} \lambda_{1} \nu_{1}=0 \\
& a_{21} \nu_{1}^{2}+b_{22} \lambda_{1} \nu_{2}=0
\end{aligned}
$$

which together with eqn (46) admit the three solutions $\nu_{1}=0, \nu_{2}=1$. $\lambda_{1}=0$ and $\nu_{1}, \pm \nu_{2}, \pm \lambda_{1}$, the first of which representing a purely local buckling mode (Fig. 13b) and the other two corresponding to interaction.

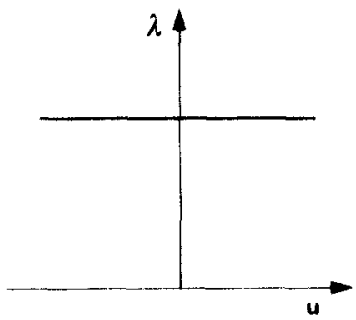

a)

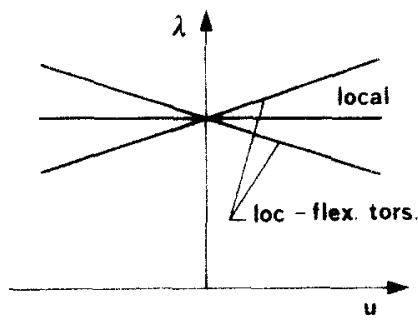

b)

Fig. 13. Local/flexural-torsional postbuckling equilibrium paths: (a) $n$ even; (b) $n$ odd. 


\section{TABLE 4}

Local/Flexural-Torsional Interaction $(\beta=0 \cdot 5)$

\begin{tabular}{rcccc}
\hline \multicolumn{1}{c}{$\alpha$} & $\gamma$ & $n$ & $\tilde{\lambda}_{1}$ & $v_{H} / w_{B}$ \\
\hline 6.47 & 0.040 & 5 & \pm 0.884 & \pm 0.352 \\
9.41 & 0.027 & 7 & \pm 0.414 & \pm 0.251 \\
11.76 & 0.022 & 9 & \pm 0.242 & $\pm 0 \cdot 195$ \\
17.25 & 0.015 & 13 & $\pm 0 \cdot 113$ & $\pm 0 \cdot 135$ \\
25.88 & 0.010 & 19 & \pm 0.052 & \pm 0.092 \\
51.76 & 0.005 & 39 & \pm 0.012 & \pm 0.045 \\
\hline
\end{tabular}

TABLE 5

Local/Flexural-Torsional Interaction $(\beta=0 \cdot 8)$

\begin{tabular}{rlccc}
\multicolumn{1}{c}{$\alpha$} & $\gamma$ & $n$ & $\tilde{\lambda}_{1}$ & $v_{H} / \boldsymbol{w}_{B}$ \\
\hline 8.68 & 0.050 & 5 & \pm 0.576 & $\pm 0 \cdot 138$ \\
12.41 & 0.035 & 7 & \pm 0.269 & \pm 0.098 \\
17.37 & 0.025 & 9 & $\pm 0 \cdot 158$ & $\pm 0 \cdot 077$ \\
$27 \cdot 14$ & 0.016 & 15 & \pm 0.055 & \pm 0.046 \\
43.42 & 0.010 & 23 & \pm 0.023 & \pm 0.030 \\
95.00 & 0.0046 & 51 & \pm 0.005 & \pm 0.014 \\
\hline
\end{tabular}

A physical explanation which accounts for the apposite sign of $\lambda_{1}$ for the interactive curves can simply be given by considering that the two different configurations corresponding to the same value of $\lambda$ can be obtained from each other through a rigid rotation of the beam around the symmetry axis of the midspan section.

For large values of $n$, the distinction between $n$ odd and even becomes meaningless and the two interactive curves are expected to approach the horizontal equilibrium path. These results do not agree with the Benito and Sridharan ${ }^{16}$ claims according to which tangents to the postbuckling equilibrium paths are always horizontal, whatever the value of $n$. Results do however coincide for large values of $n$.

A number of beams corresponding to $\beta=0.5,0.8,1.0$ have been analysed for different values of $\alpha, \gamma$ such that $\alpha \gamma=\phi_{3}(\beta)$ and are reported in Tables $4,5,6$. Obviously only beams buckling in an odd number of local halfwaves have been considered. For each value of $\beta$ it is 
TABLE 6

Local/Flexural-Torsional Interaction $(\beta=1 \cdot 0)$

\begin{tabular}{rllll}
\hline \multicolumn{1}{c}{$\gamma$} & \multicolumn{1}{c}{$n$} & \multicolumn{1}{c}{$\lambda_{i}$} & $v_{H} / w_{B}$ \\
\hline 10.87 & 0.050 & 5 & \pm 0.509 & \pm 0.097 \\
15.53 & 0.035 & 7 & \pm 0.238 & \pm 0.069 \\
19.76 & 0.0275 & 9 & \pm 0.139 & \pm 0.054 \\
37.48 & 0.0145 & 17 & \pm 0.038 & \pm 0.028 \\
54.35 & 0.010 & 25 & \pm 0.017 & \pm 0.019 \\
108.70 & 0.005 & 49 & $\pm 0 \cdot 004$ & \pm 0.010 \\
\hline
\end{tabular}

found that the two $\bar{\lambda}_{1} \mathrm{~s}$ approach zero for increasing $n$, along with the deformation parameter $v_{H} / w_{B}$, thus implying that the buckling modes tend to be purely flexural-torsional. In contrast with the results obtained for the local/eulerian interaction, it is found here that numerical results do not change drastically with $\beta$ even if this geometrical parameter ranges within a much larger interval.

In conclusion local/eulerian interaction seems to be more dangerous than the local/flexural-torsional interaction even if it occurs for a more restricted field of the geometric parameters.

\section{CONCLUSIONS}

On the basis of the general theory of elastic stability, postbuckling analysis of simultaneous buckling modes has been performed on a simply supported channel under uniform compression. A discrete model has been developed through an automatic procedure of algebraic manipulation. In the buckling analysis, fields of geometric parameters corresponding to different types of interaction have been determined and the relevant postbuckling behaviour has been investigated. Results have shown that the local/eulerian interaction is more detrimental than the local/flexural-torsional one and therefore beams exhibiting the first type of interaction are more sensitive to initial imperfections and more likely to collapse under external loads far below the critical value. A further analysis should be accomplished to evaluate the reduction of the limit load as a function of the imperfection parameters. The triple point 
$\beta=\bar{\beta}=0.3678$ of Fig. 7 corresponding to local/eulerian/flexuraltorsional interaction, also deserves investigation. Finally the analysis should be extended to cover the case of columns with symmetric postbuckling behaviour.

\section{REFERENCES}

1. Grimaldi, A. and Pignataro, M., Postbuckling behavior of thin-walled open cross-section compression members, J. Struct. Mech., 7(2) (1979) 143-59.

2. Van der Neut, A., The interaction of local buckling and column failure of thin-walled compression members, Proc. 12th Int. Congr. Appl. Mech.. Berlin, Springer-Verlag, 1969.

3. Graves-Smith, T. R., The ultimate strength of columns of arbitrary length, Symposium on thin-walled structures (Rockey, K. C. and Hill, H. V. (eds)), London, Crosby-Lockwood, 1969.

4. Graves-Smith, T. R. and Sridharan, S., A finite strip method for the post-locally-buckled analysis of plate structures, Int. J. Mech. Sci., 20 (1978), 833-42.

5. Sridharan, S. and Graves-Smith, T. R., Postbuckling analysis with finite strips, Proc. ASCE, 107(EM2) (1981) 869-87.

6. Hancock, G. J., Interaction buckling in I-section columns, J. Struct. Div., ASCE, 107(ST1) (1981) 165-79.

7. Hancock, G. J., Nonlinear analysis of thin sections in compression, $J$. Struct. Div., ASCE, 107(ST3) (1981) 455-71.

8. Bradford, M. A. and Hancock, G. J., Elastic interaction of local and lateral buckling in beams, Thin-Walled Struct., 2(1) (1984) 1-25.

9. Wang, S. T. and Pao, H. Y., Stability analysis of locally buckled singly symmetric columns, Proc. Intern. Conference on Thin-Walled Structures. Glasgow, Granada 1980, 14-27.

10. Rhodes, J. and Harvey, J. M., Interaction behaviour of plain channel columns under concentric or eccentric loading, Second Int. Colloquium on the Stability of Steel Structures, Liège, 13-15 April 1977, 439-44.

11. Loughlan, J. and Rhodes, J., The interactive buckling of lipped channel columns under concentric or eccentric loading, Proc. Intern. Conference on Thin-Walled Structures, Glasgow, Granada 1980, 40-55.

12. Loughlan, J., The ultimate load sensitivity of lipped channel columns to column axis imperfection, Thin-Walled Struct., 1(1) (1983) 75-96.

13. Upadhya, A. R. and Loughlan, J., The effect of mode interaction in orthotropic fibre reinforced composite plain channel section columns, Proc. First Int. Conf. on Composite Structures. Paisley College of Technology, Scotland, 16-18 September, 1981.

14. Loughlan, J. and Upadhya, A. R., Locally imperfect plain channel columns, In Behaviour of thin-walled structures (Rhodes, J. and Spence, J. (eds)), London, Elsevier Applied Science Publishers, 1984. 
15. Benito, R., Static and dynamic buckling of plate assemblies, PhD thesis, Washington University in St. Louis. 1983.

16. Benito, R. and Sridharan, S., Interactive buckling. A novel approach and some new results, Proc. of Third Intern. Colloq. on Stability of Metal Structures, Toronto, 1983, 91-111.

17. Sridharan, S. and Benito, R., Columns: static and dynamic interactive buckling, Proc. ASCE, 110(EM1) (1984) 49-65.

18. Koiter, W. T., Over de stabiliteit van het elastisch evenwicht (in Dutch), $\mathrm{PhD}$ thesis, H. J. Paris, Amsterdam 1945; English translation as NASA TT F-10. 833 (1967) and AFFDL Report TR 70-25 (1970).

19. Budiansky, B., Theory of buckling and post-buckling behavior of elastic structures, In Advances in applied mechanics, Vol. 14 (Chia-Shun Yih (ed.)), New York, Academic Press, 1974.

20. Van der Waerden, B. L., Modern algebra, Vol. II, New York, Ungar, 1950.

21. Vlasov, V. Z., Thin-walled elastic beams, Israel Program for Scientific Translations, Jerusalem, 1961.

22. Hearn, A. C., REDUCE-2 User's manual, University of Utah Symbolic Computation Group, Report No. UCP-19, 1973.

23. Griss, C., Using REDUCE on UNIVAC 1100, University of Utah Symbolic Computation Group, Technical Report No. TR-4, 1979.

24. Rizzi, N. and Tatone, A., Symbolic manipulation in buckling and postbuckling analysis, Istituto di Scienza delle Costruzioni, Università dell'Aquila, Report No. 71, 1983.

25. Noor, A. K., Andersen, C. M., Computerized symbolic manipulation in structural mechanics-progress and potential, Comput. Struct., 10 (1979) 95-118.

26. Noor, A. K., Balch, C. D., Hybrid perturbation/Bubnov-Galerkin technique for nonlinear thermal analysis, AIAA Journal, 22 (1984) 287-94.

27. Handbook of structural stability. Column research committee of Japan. Tokyo, Corona, 1971.

\section{APPENDIX}

The critical stresses $\sigma_{z}, \sigma_{y}, \sigma_{\theta}$, and the factor $c$ appearing in eqn (37) in terms of the geometric parameters of eqn (38) are given by the formulas

$$
\begin{aligned}
& \sigma_{z}=\frac{1}{\alpha^{2}} \mathrm{f}_{1}(\beta) \\
& \sigma_{y}=\frac{1}{\alpha^{2}} \mathrm{f}_{2}(\beta)
\end{aligned}
$$


$\sigma_{\theta}=\frac{1}{\alpha^{2} \mathrm{f}_{3}(\beta)}\left[\mathrm{f}_{4}(\beta)+\alpha^{2} \gamma^{2} \mathrm{f}_{5}(\beta)\right]$

$c=1-\frac{1}{\mathrm{f}_{3}(\beta)} 192 \beta^{4}(1+3 \beta)^{2}$

where

$$
\begin{aligned}
& \mathrm{f}_{1}(\beta)=\frac{\pi^{2}}{3} \frac{\beta^{3}(2+\beta)}{(1+2 \beta)^{2}} \\
& \mathrm{f}_{2}(\beta)=\frac{\pi^{2}}{12} \frac{1+6 \beta}{1+2 \beta} \\
& \mathrm{f}_{3}(\beta)=1872 \beta^{6}+1488 \beta^{5}+724 \beta^{4}+440 \beta^{3}+144 \beta^{2}+20 \beta+1 \\
& \mathrm{f}_{4}(\beta)=\pi^{2} \beta^{3}(2+3 \beta)(1+2 \beta)(1+6 \beta) \\
& \mathrm{f}_{5}(\beta)=\frac{2}{1+\nu}(1+6 \beta)^{2}(1+2 \beta)^{2}
\end{aligned}
$$

$\nu$ being the Poisson ratio.

Functions $\phi_{i}(\beta)(i=1,2,3)$ appearing in eqn (40) are defined by

$$
\begin{aligned}
\phi_{1}(\beta)= & \left\{\frac{\mathrm{f}_{3}}{\mathrm{f}_{5}\left(\mathrm{f}_{1}-\mathrm{f}_{2}\right)}\left[c \mathrm{f}_{1}^{2}-\mathrm{f}_{1} \mathrm{f}_{2}-\frac{\mathrm{f}_{4}}{\mathrm{f}_{3}}\left(\mathrm{f}_{1}-\mathrm{f}_{2}\right)\right]\right\}^{1 / 2} \\
\phi_{2}(\beta)= & \left(\frac{\mathrm{f}_{1}}{\eta}\right)^{1 / 2} \\
\phi_{3}(\beta)= & \left\{\left(\eta \mathrm{f}_{2} \mathrm{f}_{3}+\eta \mathrm{f}_{4}-\mathrm{f}_{2} \mathrm{f}_{5}\right)-\left[\left(\eta \mathrm{f}_{2} \mathrm{f}_{3}+\eta \mathrm{f}_{4}-\mathrm{f}_{2} \mathrm{f}_{5}\right)^{2}\right.\right. \\
& \left.\left.-4 \eta\left(c \eta \mathrm{f}_{3}-\mathrm{f}_{5}\right) \mathrm{f}_{2} \mathrm{f}_{4}\right]^{1 / 2}\right\}^{1 / 2} /\left[2 \eta\left(c \eta \mathrm{f}_{3}-\mathrm{f}_{5}\right)\right]^{1 / 2}
\end{aligned}
$$

where the argument $\beta$ of all functions has been omitted and $\eta=k \pi^{2}$ / $12\left(1-\nu^{2}\right), k$ being defined in eqn (39). 